\title{
BMJ Open Protocol of a multicentre, prospective cohort study that evaluates cost- effectiveness of two perioperative care strategies for potential obstructive sleep apnoea in morbidly obese patients undergoing bariatric surgery
}

\author{
Sophie L. van Veldhuisen (1) , ${ }^{1}$ Kim Kuppens, ${ }^{2}$ Christel A. L. de Raaff, ${ }^{3}$ \\ Marinus J. Wiezer, ${ }^{4}$ Steve M. M. de Castro, ${ }^{5}$ Ruben N. van Veen, ${ }^{5}$ \\ Dingeman J. Swank, ${ }^{6}$ Ahmet Demirkiran, ${ }^{7}$ Evert-Jan G. Boerma, \\ Jan-Willem M. Greve, ${ }^{8,9}$ Francois M. H. van Dielen, ${ }^{10}$ Geert W. J. Frederix, ${ }^{11}$ \\ Eric J. Hazebroek ${ }^{1,12}$
}

To cite: van Veldhuisen SL, Kuppens K, de Raaff CAL, et al. Protocol of a multicentre, prospective cohort study that evaluates cost-effectiveness of two perioperative care strategies for potential obstructive sleep apnoea in morbidly obese patients undergoing bariatric surgery. BMJ Open 2020;10:e038830. doi:10.1136/ bmjopen-2020-038830

- Prepublication history for this paper is available online. To view these files, please visit the journal online (http://dx.doi. org/10.1136/bmjopen-2020038830).

Received 25 March 2020 Revised 14 July 2020 Accepted 03 September 2020

D) Check for updates

(C) Author(s) (or their employer(s)) 2020. Re-use permitted under CC BY-NC. No commercial re-use. See rights and permissions. Published by BMJ.

For numbered affiliations see end of article.

\section{Correspondence to}

Sophie L. van Veldhuisen; svanveldhuisen@rijnstate.nl; s.I. vanveldhuisen@gmail.com

\section{ABSTRACT}

Introduction Despite the high prevalence of obstructive sleep apnoea (OSA) in obese patients undergoing bariatric surgery, OSA is undiagnosed in the majority of patients and thus untreated. While untreated OSA is associated with an increased risk of preoperative and postoperative complications, no evidence-based guidelines on perioperative care for these patients are available. The aim of the POPCORN study (Post-Operative Pulse oximetry without OSA sCreening vs perioperative continuous positive airway pressure (CPAP) treatment following OSA scReeNing by polygraphy (PG)) is to evaluate which perioperative strategy is the most cost-effective for obese patients undergoing bariatric surgery without a history of OSA.

Methods and analysis In this multicentre observational cohort study, data from 1380 patients who will undergo bariatric surgery will be collected. Patients will receive either postoperative care with pulse oximetry monitoring and supplemental oxygen during the first postoperative night, or care that includes preoperative PG and CPAP treatment in case of moderate or severe OSA. Local protocols for perioperative care in each participating hospital will determine into which cohort a patient is placed. The primary outcome is cost-effectiveness, which will be calculated by comparing all healthcare costs with the quality-adjusted life-years (QALYs, calculated using EQ-5D questionnaires). Secondary outcomes are mortality, complications within 30 days after surgery, readmissions, reoperations, length of stay, weight loss, generic quality of life (QOL), OSA-specific QOL, OSA symptoms and CPAP adherence. Patients will receive questionnaires before surgery and 1, 3, 6 and 12 months after surgery to report QALYs and other patient-reported outcomes.

Ethics and dissemination Approval from the Medical Research Ethics Committees United was granted in accordance with the Dutch law for Medical Research Involving Human Subjects Act (WMO) (reference number

\section{Strengths and limitations of this study}

This is the first prospective study to compare continuous postoperative pulse oximetry without preoperative obstructive sleep apnoea (OSA) screening to routine OSA screening with consequent continuous positive airway pressure (CPAP) treatment as perioperative care for bariatric patients with potential OSA.

- The main outcome of this study is cost-effectiveness of these two perioperative care strategies in obese patients with no known OSA history.

- Results of this study will provide new insights in unknown aspects of perioperative interventions for undetected OSA, such as the impact on postoperative complications and general quality of life (QOL).

- Despite the non-randomised design, we hypothesise that the results are generalisable to most bariatric centres due to the large sample size and limited exclusion criteria.

- Our follow-up duration of 1 year enables analysis of long-term outcomes of perioperative interventions for OSA, such as influence on weight loss, sleepiness symptoms, QOL with sleepiness and adherence to CPAP.

W17.050). Results will be submitted for publication in peer-reviewed journals and presented at (inter)national conferences.

Trial registration number NTR6991.

\section{INTRODUCTION}

Obesity is a healthcare issue of epidemic proportions that is rapidly increasing. Worldwide, more than 650 million people are affected by obesity, defined as body mass 
index (BMI) $\geq 30 \mathrm{~kg} / \mathrm{m}^{2}$, with subsequent morbidity and mortality. ${ }^{1}$ Many conservative and lifestyle interventions that are aimed at reducing weight are available but most lack effectiveness and durable results. To date, bariatric surgery is the only effective treatment for obesity that achieves sustainable, long-term weight loss. ${ }^{23}$

Obesity is the main risk factor for obstructive sleep apnoea (OSA), a sleep-breathing disorder with recurrent breathing cessations that occur when the pharyngeal airway collapses completely or partially. These collapses are respectively called apnoeas and hypopnoeas. The number of breathing cessations per hour of sleep, the apnoea hypopnoea index (AHI), indicates the severity of OSA. ${ }^{45}$ Intermittent hypoxaemia, hypercapnia and arousals from sleep are a result of breathing cessations, which lead to excessive daytime sleepiness, cognitive impairment and increased risk of cardiovascular disease. The golden standard for OSA diagnosis is an in-laboratory polysomnography (PSG), but in recent years, homebased polygraphy (PG) has also been validated as a diagnostic tool. ${ }^{6}$ Currently, the best treatment for OSA is positive airway pressure (PAP), most commonly provided as continuous PAP (CPAP), and aims to maintain an open airway during sleep. Hereby, arousals from sleep will be reduced, which improves daytime functioning with less excessive sleepiness, as well as quality of life (QOL) and cognitive functioning. ${ }^{7}$

OSA is highly prevalent in patients who are eligible for bariatric surgery, affecting approximately 60\%-70\%, compared with OSA prevalence of $3 \%-17 \%$ in the general adult population. ${ }^{8-10}$ Due to the strong correlation of OSA and obesity, weight loss should be recommended to all obese patients with moderate or severe OSA. ${ }^{11}{ }^{12}$ Bariatric surgery is highly effective for this disease, as $60 \%-85 \%$ patients achieve complete remission of OSA or significant reduction of their disease severity. ${ }^{23-16}$

Perioperative care for bariatric patients with OSA pose a clinical challenge, given that the majority is asymptomatic or experiences unrecognised symptoms and is consequently untreated. ${ }^{17}$ Opioids administered during general anaesthesia can induce long-lasting apnoeas in patients with untreated OSA. As a result, (untreated) OSA is associated with a higher risk of cardiopulmonary and neurovascular complications, as well as higher overall mortality and morbidity in general surgery populations. ${ }^{1819}$ Evidence that this phenomenon of increased perioperative risk also exists in bariatric patients is thin, and most studies do not mention whether precautions were taken to prevent OSA-related adverse events. ${ }^{20}$ More recent prospective studies and reviews demonstrate a consistently low incidence of cardiopulmonary and neurovascular complications after bariatric surgery, and statistical analyses fail to indicate a direct causative link to OSA. ${ }^{21-23}$

Evidence-based guidelines for perioperative care of potential OSA in bariatric patients are lacking. ${ }^{24}$ Therefore, a wide variety of perioperative modalities has emerged, which all aim to minimise the risk of serious adverse events related to untreated OSA. One of the options is routine preoperative assessment of OSA in every bariatric patient by performing PSG or PG. Newly diagnosed moderate or severe OSA patients will consequently be treated with CPAP. Another option relies on questionnaires to identify patients at high risk of OSA who subsequently undergo PG. These questionnaires, such as the STOP-BANG or Berlin questionnaire, are frequently used, but none of these screening tools has been able to render both high sensitivity and specificity. Therefore, its applicability remains controversial. ${ }^{25-27}$ Another alternative is routine, postoperative continuous monitoring with pulse oximetry with supplemental non-invasive oxygen administration but without preoperative OSA assessment. In this approach, all patients receive the same intervention to achieve adequate saturation levels in the early postoperative phase. $^{21}$

Obesity and obesity-related disorders increasingly demand utilisation of available healthcare resources. Justification of high screening expenses for OSA is debatable given the low incidence of OSA-related complications, despite the high prevalence of OSA. In addition, CPAP adherence rates are poor even in patients with symptomatic OSA, ranging between $29 \%$ and $83 \% .{ }^{28}$ While specific data are lacking, adherence rates are putatively even lower in asymptomatic bariatric patients, which questions the actual protective effect that is added by preoperative initiation of CPAP. In contrast, adequate treatment with CPAP in symptomatic OSA patients positively influences societal costs, as symptomatic patients without treatment use more healthcare resources, suffer more unemployment and are more prone to work-related or traffic accidents. ${ }^{29-31}$ However, routine screening and treatment of asymptomatic patients is not likewise supported by conclusive evidence. ${ }^{27}{ }^{32}$ Deliberate consideration is needed when comparing outcomes such as safety, costs and patients' satisfaction between different perioperative strategies for OSA care in bariatric patients.

\section{RATIONALE}

The primary aim of the POPCORN study (Post-Operative Pulse oximetry without OSA sCreening vs OSA scReeNing) is to evaluate the most cost-effective perioperative strategy for bariatric patients who have no history of OSA. We will compare postoperative continuous pulse oximetry without OSA screening with routine OSA screening by PG and subsequent application of CPAP. This study will provide evidence that will enable clinicians to make an evidence-based decision on perioperative care of patients with no known OSA undergoing bariatric surgery. This paper describes the design and protocol of the POPCORN study.

\section{METHODS AND ANALYSIS \\ Study design}

The POPCORN study is a prospective, multicentre, observational cohort study that evaluates two cohorts of bariatric patients who have no history of OSA. The first cohort consists of patients who are postoperatively monitored with 
Pre-operative

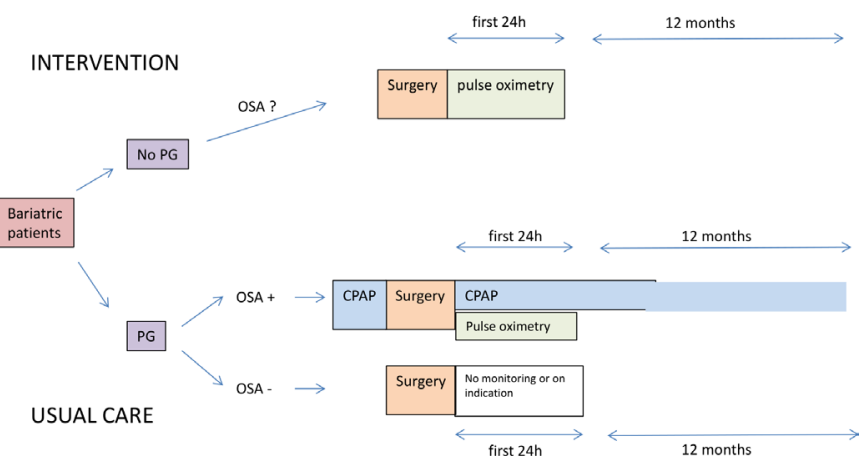

Figure 1 Flowchart of the POPCORN study (Post-Operative Pulse oximetry without OSA sCreeningvs. OSA scReeNing). CPAP, continuous positive airway pressure; PG, polygraphy; OSA, obstructive sleep apnoea.

continuous pulse oximetry (CPOX cohort) who do not undergo a PG or a PSG. In the second cohort, all bariatric patients undergo a preoperative PG and in case of moderate or severe OSA receive consequent treatment with CPAP before and after surgery (preoperative polygraphy (PPG) cohort) (figure 1).

\section{Recruitment procedures and consent}

In total, 1380 obese patients scheduled to undergo bariatric surgery will be included for participation in the POPCORN study. For study participation, a subject must meet the following inclusion criteria: (1) preoperative BMI $\geq 35 \mathrm{~kg} / \mathrm{m}^{2}$ combined with an obesity-related comorbidity or preoperative $\mathrm{BMI} \geq 40 \mathrm{~kg} / \mathrm{m}^{2},{ }^{33}$ (2) age $\geq 18$ years, (3) undergo a primary bariatric procedure. Potential subjects will be excluded from participating in the following situations: (1) previous bariatric surgery, such as laparoscopic adjustable gastric banding; (2) inability to speak or read the Dutch language; (3) concomitantly performed procedures during bariatric surgery that increase the risk of postoperative complications and costs, such as cholecystectomy or paraoesophageal hernia repair; (4) use of treatment options for OSA other than PAP modalities, such as a mandibular advancement device or positional therapy.

In both cohorts, 690 patients will be included. Local protocols of participating hospitals will determine which strategy of perioperative care is used and this will consequently determine the allocation of patients into one of the two cohorts. Seven hospitals in the Netherlands will collaborate to recruit all study patients. Of the participating hospitals, the only hospital that applies CPOX without preoperative OSA screening is Rijnstate Hospital, Arnhem, which will recruit patients for the CPOX arm. For the PG cohort, patients are recruited from the other participating hospitals (St. Antonius Hospital, Nieuwegein; Onze Lieve Vrouwe Hospital, Amsterdam; Dutch Obesity Clinic, the Hague; Zuyderland Hospital, Heerlen; Rode Kruis Hospital, Beverwijk and Máxima Medical Centre, Veldhoven). Written or digitally signed informed consent will be obtained from all participants enrolled in this study. Recruitment has started in April 2018 and is expected to be completed in March 2020.

\section{CPOX cohort}

Bariatric patients in the CPOX cohort receive no preoperative screening for OSA: no PG, polysomnography or questionnaires for risk stratification are conducted. Postoperatively, bariatric patients return to the surgical ward where continuous surveillance with pulse oximetry is immediately started, with supplemental oxygen provided via a nasal cannula $\left(2 \mathrm{~L} / \mathrm{min} \mathrm{SpO}_{2}\right)$. Pulse oximetry is performed using a Draeger Infinity Delta monitor (Draeger Medical Systems Incorporated, USA). Clinical desaturations are defined as $<92 \% \mathrm{SpO}_{2}$, lasting at least $10 \mathrm{~s}$. A desaturation sets off an alarm that alerts the attending nurse who will perform a clinical evaluation. Long-lasting apnoeas can either be terminated by awaking the respective patient, or by providing additional supplemental oxygen via the non-invasive nasal cannula. In case of a serious desaturation that cannot be managed appropriately by these minor interventions, patients can be admitted to the intensive care unit (ICU) for potential reintubation at discretion of the treating physician.

\section{PPG cohort}

The PPG cohort will consist of bariatric patients that are preoperatively screened for OSA with a polygraphy (PG) or polysomnography (PSG). In patients with moderate or severe disease, defined as $\mathrm{AHI} \geq 15$ and $\mathrm{AHI} \geq 30$ events/ hour, CPAP treatment is initiated. In patients with mild disease, defined as AHI 5-14 events/hour, CPAP is only advised in the presence of clinically significant symptoms such as excessive sleepiness and unrefreshing sleep. ${ }^{34}$ In patients where an AHI of $<5$ events/hour is observed, OSA is excluded and no additional perioperative precautions are needed (figure 1). In mild, moderate and severe disease, automatic or bi-level continuous airway pressure (APAP and BiPAP) are considered qualitatively equal, compared with CPAP. Therefore, if CPAP treatment is unsuccessful, APAP and BiPAP are also defined as optimal treatment in the perioperative phase.

\section{Enhanced recovery after bariatric surgery protocols}

All participating hospitals will use preoperative and postoperative protocols during the study period that are based on the principles of Enhanced Recovery After Bariatric Surgery (ERABS) ${ }^{35}$ These principles underline aspects of care that enable quick recovery after surgery to minimise preoperative and postoperative opioid administration and to stimulate early postoperative mobilisation. To prepare patients for the bariatric procedure and the associated lifestyle changes, all centres have comparable preoperative and postoperative programmes for bariatric care. This enlarges a patients' knowledge and expectations on the procedure, the admission and alarm signs for adverse events. 


\section{Surgical procedures}

Laparoscopic Roux-en-Y gastric bypass (LRYGB) is the most performed procedure in all participating hospitals, followed by laparoscopic sleeve gastrectomy (LSG). LRYGB and LSG are both stomach-reducing procedures, and thus induce significant restriction on food intake. Both procedures influence metabolic and hormonal responses that additionally contribute to weight loss. Furthermore, LRYGB has an additional malabsorptive element as food bypasses the duodenum and a part of the ileum. Both procedures are performed in a protocolled fashion and will be very similar in all participating hospitals.

\section{Primary outcomes}

Cost-effectiveness of CPOX compared with standard care with PPG is the primary outcome and will be evaluated during the period from baseline to 12 months after surgery from a societal perspective. Effectiveness of perioperative care (eg, CPOX and PPG) will be expressed in qualityadjusted life-years (QALYs). The QALYs will be calculated using the EuroQol 5 Dimensions-3 level (EQ-5D-3L) questionnaire, which rates a person's autonomy and well-being on five scales; mobility, self-care, usual activities, pain/discomfort and anxiety/depression. ${ }^{36}$ All scores will be calculated using the subset that was validated for the Dutch population of the EQ-5D-3L. ${ }^{37}$ Additionally, patients indicate their general health of that day on a Visual Analogue Scale (VAS). The EQ-5D score creates a so-called utility between 0 and 1 , indicating 1 as the highest form of well-being and 0 as the lowest form of well-being, that is, death.

Direct and indirect costs during the entire study period will be assessed for each individual study subject. Direct costs will be extracted from hospital files and electronic patient records. These costs will be carefully evaluated with regard to the relationship with obesity or OSA. Any unrelated costs will not be considered for the cost-effectiveness analysis. Uncertainty regarding the involvement of OSA or obesity on certain healthcare costs will be resolved by discussion between authors SLvV, EJH and KK.

In addition, we aim to collect healthcare costs outside the hospital and so-called indirect costs which refer to lost resources and opportunities (for instance inability to work) resulting from OSA. These costs will be evaluated using two questionnaires: the Productivity Costs Questionnaire (PCQ) and the Medical Costs Questionnaire (MCQ). The PCQ is a validated questionnaire that assesses the relationship of general income and productivity to physical and mental wellbeing. ${ }^{38}$ The MCQ is used to measure extramural medical costs, for example, visits to a general practitioner or a dietician, or medical care in another hospital than the bariatric centre. The PCQ and MCQ questionnaires are conducted at 3 and 12 months, postoperatively.

\section{Secondary outcomes}

Mortality, morbidity, complications, ICU admissions, length of hospital stay, OSA-related symptoms, adherence to CPAP and QOL are all secondary outcomes.

Baseline morbidity will be documented and remission of OSA evaluated after 12 months in the patient files, for example, comorbidities resolution and weight loss progression during the first postoperative year. Weight loss will be expressed as percentage excessive weight loss (\% EWL), percentage total weight loss (\% TWL) and change in BMI.

\section{Complications}

All complications that occur within 30 days of the bariatric procedure will be analysed.

Distinction will be made in each complication whether it could be caused by (untreated) OSA; this will mainly entail pulmonary, cardiac, thromboembolic and neurovascular complications. Uncertainty regarding these decisions will be solved by discussion between authors SLvV, $\mathrm{EJH}$ and KK. If the authors conclude that a pulmonary, cardiac, thromboembolic or neurovascular complication is not a result of OSA, this will be described in the manuscript. Severity of complications will be registered according to the Clavien-Dindo Classification. ${ }^{39}$

\section{Quality of life}

Generic QOL will be measured using the EQ-5D-3L, and the Rand-Short Form 36-items questionnaire, which assesses general health in nine different aspects, including physical activity and bodily pain. ${ }^{40}$ Sleep-related QOL will be assessed with the Functional Outcome Sleep Questionnaire-10. ${ }^{41}$ This 10-item questionnaire measures the effect of tiredness and sleepiness on QOL, and scores are obtained through a 4-point Likert scale. The outcome score ranges from 5 to 20: low scores indicate poor QOL that is greatly influenced by daytime sleepiness, while high scores inversely indicate good QOL uninfluenced by daytime sleepiness. $^{42}$

\section{OSA-related outcomes}

The main symptom of OSA, daytime sleepiness, will be assessed by the Epworth Sleepiness Scale questionnaire. ${ }^{43}$ Patients report the likelihood of falling asleep during eight daytime activities on a Likert scale of 0-3, indicating results that range from normal daytime sleepiness (score 0-5) to severe excessive daytime sleepiness (score 16-24).

Preoperative and postoperative PGs (or PSGs) during the study period will be analysed for AHI, AHI in supine position, oxygen desaturation index, total sleeping time in supine position, mean oxygen saturation, lowest oxygen saturation, time of saturation $<90 \% \quad \mathrm{SpO}_{2}$, number of episodes of saturation $<90 \% \mathrm{SpO}_{2}$ and number of episodes with $>4 \%$ saturation drop below mean saturation. Additional factors that could contribute to disease-load or probability are also monitored; previous ENT surgery that provides a wider pharyngeal girth (ie, uvulopalatopharyngoplasty), smoking status, alcohol consumption and daily use of opioids and benzodiazepines will also be registered.

\section{CPAP adherence}

Due to known discrepancies between patient-reported adherence to treatment and objective treatment adherence data, we will obtain both objective and subjective 
data on CPAP adherence. Adherence will be expressed in days per week of CPAP treatment and hours per night. To obtain objective data, we will consult online databanks for collection of day-to-day adherence rates. CPAP devices automatically send adherence data and corresponding AHIs to an online databank, which healthcare providers in the Netherlands use to monitor their patients. In addition, electronic patient records will be evaluated for physicians' recommendation regarding (dis)continuation of CPAP during follow-up.

Subjective data on CPAP adherence will be collected through patient-reported outcomes measurements. By using questionnaires, insight can be obtained regarding patients' motives for treatment discontinuation.

\section{Data management}

Handling of data was prospectively addressed in a data management plan with the aim of generating data in accordance with the FAIR criteria: Findable, Accessible, Intraoperable and Reusable.

\section{Sample size calculation}

A non-inferiority design was chosen to evaluate whether CPOX with no preoperative PG is non-inferior to preoperative PG in bariatric patients. In patients with moderate or severe OSA, CPAP treatment is part of standard care. The primary outcome is QALY difference compared with costs, where QALYs are measured by the EQ-5D. Therefore, the sample size calculation is based on a predefined non-inferiority margin of 0.03 on the EQ-5D score. Based on an EQ-5D score of 0.68 in the usual care group, QALYs of patients with OSA before and after 1 year of CPAP treatment, and calculating with $80 \%$ power to detect the predefined non-inferiority margin at a one sided a level of 0.05 , there are 621 patients needed in each study group. ${ }^{44}$ Assuming a loss to follow-up of $10 \%$, the total study population will be set at 1380 patients, resulting in 690 patients per arm.

\section{Analysis of primary outcome measures}

An extensive cost-effectiveness analysis (CEA) and budget impact analysis (BIA) will be performed. The CEA adheres to the Dutch guideline ${ }^{45}$ and reporting will adhere to the CHEERS checklist. ${ }^{46}$ The BIA will adhere to current Dutch guidelines and also guidelines as published by Sullivan $e$ e l. $^{47}$ We aim to perform a trial-based economic evaluation in which we do not extrapolate costs and effect outside the study period. The effect of the CEA will be expressed in change of QALYs during the study period, and this outcome will be compared with the total costs of each individual patient. Outcomes will be average cost per patient, differences between groups and incremental costs per QALY. An incremental cost-effectiveness ratio analysis will be performed to compare the outcomes (in QALY) rendered by the CPOX and the PPG strategy to the costs related to each perioperative strategy.

Sensitivity analysis will be carried out to correct all potential confounders, such as gender, age, preoperative
BMI, comorbidities, choice of bariatric centre, intraoperative and postoperative administered opioids, smoking status, previous ear, nose and throat (ENT) surgery. Oneway sensitivity analyses will be illustrated graphically using tornado diagrams; probabilistic sensitivity analyses will be illustrated in cost-effectiveness planes and so-called costeffectiveness acceptability curves. Bootstrapping will be used if deemed necessary.

\section{Cost assessment}

This analysis will be performed using a societal perspective.

- Identification: we aim to identify all healthcare utilisation for every included patient within the study period. All consumption potentially related to obesity, bariatric surgery and obstructive sleep apnoea will be identified in this total set of healthcare consumption. The latter comprised a fast amount of healthcare resources that are potentially related to OSA: cost resources related to sleep medicine, cardiovascular disease, pulmonary disease, ENT disease, and workrelated or traffic-related accidents.

- Measurement: utilisation of healthcare resources within the hospital were gathered by using each hospital billing system (detailed healthcare consumption data send to insurance companies). Additional medical costs that were made in a different hospital or outside of hospitals (ie, visits to the general practitioner, dietician, physical therapist) were scored based on patients' answers in the MCQ. The outcomes were scored in a numerical manner, for example, 0 , 1,2 visits to the GP. These results were analysed and valued based on a fixed national cost as documented in the Dutch Healthcare Institute guideline.

- Evaluation of costs: unit costs used are derived from the guidelines commissioned by the Dutch Healthcare Institute (Zorginstituut Nederland). Moreover, additional unit costs are gathered from the Dutch Healthcare Authority (Nederlandse Zorg Autoriteit; https://www.nza.nl/).

\section{Analysis of secondary outcome measures}

Baseline characteristics of patients will be documented with mean/SD or median/range, depending on normality. The number of desaturations, both cardiopulmonary and general complications, interventions, total hospital stay and total costs between both groups will be analysed with the independent t-test/Mann-Whitney $\mathrm{U}$ test. Compliance of CPAP and opioids use will be evaluated with $\chi^{2}$ testing. Mixed model analysis will be performed to evaluate the weight loss, severity of OSA symptoms and CPAP adherence at different time points. Predictive values for cardiopulmonary complications will be evaluated with logistic regression analysis, starting with a univariate analysis. All variables with a significance level $\mathrm{p}<0.2$ will be included in a multivariate analysis. Within this analysis, only seven independent variables may be included as 10 event cases are allowed per dependent predictor. Statistical significance is defined as $\mathrm{p}<0.05$. 
To correct for potential confounder between these (non-randomised) cohorts, all outcomes will be analysed by propensity score matching or multivariate analysis, depending on the secondary outcome of interest. ${ }^{48}$

\section{Loss to follow-up or replacement of participants}

Study participants will be replaced with new participants in case of (1) cancellation of the surgery, (2) uncompleted preoperative questionnaire or (3) when PAP treatment is switched to a different modality such as a mandibular advancement device. Patients who do not complete the postoperative questionnaire at 12 months after surgery due to other reasons will be considered lost to follow-up.

\section{Patient and public involvement}

Patients and the public were not involved in the design, or conduct, of our research. However, a non-profit organisation for patients with OSA was consulted in the final phase of designing this study. The organisation underlined the need for this research and requested no significant changes to the protocol. In addition, patients with OSA who previously underwent bariatric surgery in the hospital that initiated this study were invited to share their opinion on the questionnaires and OSA outcomes.

\section{Ethics and dissemination}

The Medical Ethics Committee United (MEC-U) approved this study, in accordance with the Dutch law Medical Research Involving Human Subjects Act (WMO), Medical Research in Humans (MEC-U, W17.050). In addition, local Medical Ethics Committees of each participating hospital also reviewed and approved the study protocol.

Findings of the POPCORN study will be disseminated to all disciplines that are involved in care for bariatric surgery, through articles in peer-reviewed journals, national and international congresses, and revising the national guidelines of the Netherlands.

\section{DISCUSSION}

The POPCORN study is a prospective observational cohort study that evaluates the cost-effectiveness of two strategies of perioperative care in bariatric patients without a preexistent OSA diagnosis: CPOX without extensive preoperative OSA screening versus mandatory PG, potentially followed by CPAP treatment. The outcomes will enable the development of new, evidence-based guidelines on perioperative care for bariatric patients with no known OSA. The secondary outcomes, such as (cardiopulmonary) complications, OSA-related symptoms and QOL, will provide an overview of the correlation between costeffectiveness and clinical outcomes that are highly relevant in the decision-making for perioperative care in bariatric patients.

Best practice regarding perioperative care in bariatric patient has been an ongoing debate for many years, with high prevalence and potential detrimental effects of undetected
OSA on one side and substantial costs of related perioperative care and CPAP treatment on the other. ${ }^{27} 4950$ No comparative studies between different perioperative strategies have been conducted to evaluate outcomes of postoperative complications or cost-effectiveness. In a recent review, conducted by the US preventative task force, no effectiveness of OSA screening in patients who are asymptomatic or who experience unrecognised symptoms was found. ${ }^{27}$ Despite improvements in intermediate outcomes such as AHI or sleepiness symptoms, no improvement in final health outcomes have been demonstrated, such as mortality or serious adverse events. The paucity in evidence regarding beneficial outcomes is especially relevant when cost-effectiveness is regarded. The obesity epidemic and its related costs are continuously expanding, and this underlines the need for optimal use of available healthcare resources. With no confirmative data on positive influence of OSA screening in bariatric patients with no known OSA, and approximately 700000 bariatric procedures annually worldwide, clarification on this topic is needed.

The perioperative strategies evaluated in the POPCORN study are both widely used in general practice and it is expected that results of this study will lead to evidencebased recommendations and guidelines.

The strength of this study is that a general, bariatric population is evaluated. In both cohorts, large groups of bariatric patients are prospectively observed, while little exclusion criteria are applied. In addition, the follow-up period in this study that investigates a perioperative intervention is relatively long. Previous studies that describe preoperative assessment and treatment of OSA mainly reported prevalence of newly detected OSA and related adverse outcomes restricted to the direct perioperative period. $^{51}$ The follow-up duration of 1 year after surgery enables us to investigate long-term clinical outcomes of a perioperative regime. Interesting comparisons are to be made between the preoperatively diagnosed OSA patients and the unscreened bariatric patients in terms of sleepiness symptoms, daytime productivity, general QOL and healthcare resource utilisation.

Ideally, a randomised controlled trial would have been conducted, in which all patients would undergo a preoperative PG. Consecutive randomisation would have determined the type of perioperative care: CPOX monitoring or treatment based on the PG outcome. However, this was considered unethical, as randomisation into the CPOX cohort would result in withholding appropriate treatment from patients with confirmed OSA diagnosis, which is associated with many healthcare hazards. ${ }^{30} 3152$ Despite the non-randomised design of the POPCORN trial, the large sample size will provide sufficient data to render a balanced statement that will be representable for the general bariatric population in the Netherlands. Furthermore, it is expected that implementation of these perioperative strategies is also feasible in countries other than the Netherlands.

In addition to the non-randomised design of the POPCORN study, another limitation is that preoperative 
weight loss programmes can result in changes in comorbidities. This can be particularly true for OSA, a comorbidity that is greatly influenced by weight loss. Each participating hospital applied local protocols that advocate weight loss before surgery, but in the absence of a strict programme, we do not expect major changes in weight between preoperative (OSA) assessment and the surgical procedure. In conclusion, the POPCORN study will conclude which perioperative strategy is the most cost-effective for obese patients scheduled for bariatric surgery and who have an unknown OSA status. These data will contribute to evidence-based guidelines that are urgently needed in this particular field of bariatric care.

\section{Author affiliations}

${ }^{1}$ Department of Surgery / Vitalys Clinic, Rijnstate Ziekenhuis, Arnhem, The Netherlands

${ }^{2}$ Department of Pulmonary Medicine, St. Antonius Ziekenhuis, Nieuwegein, The Netherlands

${ }^{3}$ Department of Surgery, Albert Schweitzer Ziekenhuis, Dordrecht, The Netherlands ${ }^{4}$ Department of Surgery, St. Antonius Ziekenhuis, Nieuwegein, The Netherlands ${ }^{5}$ Department of Surgery, OLVG, location West, Amsterdam, The Netherlands ${ }^{6}$ Department of Surgery, Dutch Obesity Clinic (Nederlandse Obesitas Kliniek), The Hague, The Netherlands

${ }^{7}$ Department of Surgery, Rode Kruis Ziekenhuis, Beverwijk, The Netherlands ${ }^{8}$ Department of Surgery, Zuyderland Medisch Centrum, Heerlen, The Netherlands ${ }^{9}$ Department of Surger / Nutrim, Maastricht University, Maastricht, The Netherlands ${ }^{10}$ Department of Surgery, Maxima Medisch Centrum, Eindhoven, The Netherlands

${ }^{11}$ Department of Public Health, Julius Center Research Program Methodology, Universitair Medisch Centrum Utrecht, Utrecht, The Netherlands

${ }^{12}$ Division of Human Nutrition and Health, Wageningen University and Research, Wageningen, Gelderland, The Netherlands

Contributors EJH conceived and designed the study, with support of MJW, SMMdC, RNvV, DJS, AD, E-JGB, J-WMG and FMHvD, who all contributed significantly to the study design. CALdR, KK and GWJF reviewed and refined the research questions and search strategy. SLvV wrote the first draft of this manuscript and developed the protocol together with EJH, CALdR, KK, GWJF and LR. EJH is the principal investigator and SLvV is the main investigator. All authors critically reviewed the content and approved the final manuscript.

Funding This work was supported by ZonMw, grant number '843004110'. Competing interests None declared.

Patient consent for publication Not required.

Provenance and peer review Not commissioned; externally peer reviewed.

Open access This is an open access article distributed in accordance with the Creative Commons Attribution Non Commercial (CC BY-NC 4.0) license, which permits others to distribute, remix, adapt, build upon this work non-commercially, and license their derivative works on different terms, provided the original work is properly cited, appropriate credit is given, any changes made indicated, and the use is non-commercial. See: http://creativecommons.org/licenses/by-nc/4.0/.

ORCID iD

Sophie L. van Veldhuisen http://orcid.org/0000-0002-0044-8441

\section{REFERENCES}

1 WHO. Obesity and overweight; fact sheet, 2018.

2 Buchwald $\mathrm{H}$, Avidor $\mathrm{Y}$, Braunwald $\mathrm{E}$, et al. Bariatric surgery: a systematic review and meta-analysis. JAMA 2004;292:1724-37.

3 Maciejewski ML, Arterburn DE, Van Scoyoc L, et al. Bariatric surgery and long-term durability of weight loss. JAMA Surg 2016;151:1046-55.

4 Young T, Skatrud J, Peppard PE. Risk factors for obstructive sleep apnea in adults. JAMA 2004;291:2013-6.

5 Peppard PE, Young T, Palta M, et al. Longitudinal study of moderate weight change and sleep-disordered breathing. JAMA 2000;284:3015-21.
6 Sateia MJ. International classification of sleep disorders-third edition: highlights and modifications. Chest 2014;146:1387-94.

7 Giles TL, Lasserson TJ, Smith BJ, et al. Continuous positive airways pressure for obstructive sleep apnoea in adults. Cochrane Database Syst Rev 2006;25.

8 de Raaff CAL, Pierik AS, Coblijn UK, et al. Value of routine polysomnography in bariatric surgery. Surg Endosc 2017;31:245-8.

9 Peromaa-Haavisto P, Tuomilehto H, Kössi J, et al. Prevalence of obstructive sleep apnoea among patients admitted for bariatric surgery. A prospective multicentre trial. Obes Surg 2016;26:1384-90.

10 Peppard PE, Young T, Barnet JH, et al. Increased prevalence of sleep-disordered breathing in adults. Am J Epidemiol 2013;177:1006-14.

11 Hudgel DW, Patel SR, Ahasic AM, et al. The role of weight management in the treatment of adult obstructive sleep apnea. An official American thoracic Society clinical practice guideline. Am J Respir Crit Care Med 2018;198:e70-87.

12 Young T, Peppard PE, Gottlieb DJ. Epidemiology of obstructive sleep apnea: a population health perspective. Am J Respir Crit Care Med 2002;165:1217-39.

13 Sarkhosh K, Switzer NJ, El-Hadi M, et al. The impact of bariatric surgery on obstructive sleep apnea: a systematic review. Obes Surg 2013;23:414-23.

14 Ravesloot MJL, Hilgevoord AAJ, van Wagensveld BA, et al. Assessment of the effect of bariatric surgery on obstructive sleep apnea at two postoperative intervals. Obes Surg 2014;24:22-31.

15 de Raaff CAL, Coblijn UK, Ravesloot MJL, et al. Persistent moderate or severe obstructive sleep apnea after laparoscopic Roux-en-Y gastric bypass: which patients? Surg Obes Relat Dis 2016;12:1866-72.

16 van Veldhuisen SL, Hazebroek EJ. Obstructive sleep apnea in adults. N Engl J Med 2019;381:e7.

17 Singh M, Liao P, Kobah S, et al. Proportion of surgical patients with undiagnosed obstructive sleep apnoea. Br J Anaesth 2013;110:629-36.

18 Kaw R, Michota F, Jaffer A, et al. Unrecognized sleep apnea in the surgical patient: implications for the perioperative setting. Chest 2006;129:198-205.

19 Kaw R, Chung F, Pasupuleti V, et al. Meta-analysis of the association between obstructive sleep apnoea and postoperative outcome. $\mathrm{Br} \mathrm{J}$ Anaesth 2012;109:897-906.

20 Flancbaum L, Belsley S. Factors affecting morbidity and mortality of Roux-en-Y gastric bypass for clinically severe obesity: an analysis of 1,000 consecutive open cases by a single surgeon. $J$ Gastrointest Surg 2007;11:500-7.

21 van Veldhuisen SL, Arslan I, Deden LN, et al. Safety of continuous postoperative pulse oximetry monitoring without obstructive sleep apnea screening in $>5000$ patients undergoing bariatric surgery. Obes Surg 2019

22 O'Reilly E, Doherty L, O'Boyle C. How relevant is pre-operative obstructive sleep apnoea in the asymptomatic bariatric surgery patient? Obes Surg 2019

23 Meurgey J-H, Brown R, Woroszyl-Chrusciel A, et al. Peri-operative treatment of sleep-disordered breathing and outcomes in bariatric patients. J Thorac Dis 2018;10:S144-52.

24 de Raaff CAL, Gorter-Stam MAW, de Vries N, et al. Perioperative management of obstructive sleep apnea in bariatric surgery: a consensus guideline. Surg Obes Relat Dis 2017;13:1095-109.

25 Chiu H-Y, Chen P-Y, Chuang L-P, et al. Diagnostic accuracy of the Berlin questionnaire, Stop-Bang, stop, and Epworth Sleepiness scale in detecting obstructive sleep apnea: a bivariate meta-analysis. Sleep Med Rev 2017;36:57-70.

26 Turnbull CD, Stradling JR. To screen or not to screen for obstructive sleep apnea, that is the question. Sleep Med Rev 2017;36:125-7.

27 US Preventive Services Task Force, Bibbins-Domingo K, Grossman DC, et al. Screening for obstructive sleep apnea in adults: US preventive services Task force recommendation statement. JAMA 2017;317:407-14.

28 Weaver TE, Grunstein RR. Adherence to continuous positive airway pressure therapy: the challenge to effective treatment. Proc Am Thorac Soc 2008;5:173-8.

29 Tregear S, Reston J, Schoelles K, et al. Continuous positive airway pressure reduces risk of motor vehicle crash among drivers with obstructive sleep apnea: systematic review and meta-analysis. Sleep 2010;33:1373-80.

30 Somers VK, White DP, Amin R, et al. Sleep apnea and cardiovascular disease: an American heart Association/American College of cardiology Foundation scientific statement from the American heart association Council for high blood pressure research professional education Committee, Council on clinical cardiology, stroke 
Council, and Council on cardiovascular nursing. J Am Coll Cardiol 2008;52:686-717.

31 Siccoli MM, Pepperell JCT, Kohler M, et al. Effects of continuous positive airway pressure on quality of life in patients with moderate to severe obstructive sleep apnea: data from a randomized controlled trial. Sleep 2008;31:1551-8.

32 Jonas DE, Amick HR, Feltner C, et al. Screening for obstructive sleep apnea in adults: evidence report and systematic review for the US preventive services Task force. JAMA 2017;317:415-33.

33 Fried M, Hainer V, Basdevant A, et al. Interdisciplinary European guidelines on surgery of severe obesity. Obes Facts 2008;1:52-9.

34 Epstein LJ, Kristo D, Strollo PJ, et al. Clinical guideline for the evaluation, management and long-term care of obstructive sleep apnea in adults. J Clin Sleep Med 2009;5:263-76.

35 Thorell A, MacCormick AD, Awad S, et al. Guidelines for perioperative care in bariatric surgery: enhanced recovery after surgery (ERAS) Society recommendations. World J Surg 2016;40:2065-83.

36 Brooks R. EuroQol: the current state of play. Health Policy 1996;37:53-72.

37 Lamers LM, McDonnell J, Stalmeier PFM, et al. The Dutch tariff: results and arguments for an effective design for national EQ-5D valuation studies. Health Econ 2006;15:1121-32.

38 Bouwmans $\mathrm{C}$, Krol M, Severens $\mathrm{H}$, et al. The iMTA productivity cost questionnaire: a standardized instrument for measuring and Valuing health-related productivity losses. Value Health 2015;18:753-8.

39 Dindo D, Demartines N, Clavien P-A. Classification of surgical complications: a new proposal with evaluation in a cohort of 6336 patients and results of a survey. Ann Surg 2004;240:205-13.

40 Brazier J, Roberts J, Deverill M. The estimation of a preferencebased measure of health from the SF-36. J Health Econ 2002;21:271-92.

41 Chasens ER, Ratcliffe SJ, Weaver TE. Development of the FOSQ-10: a short version of the functional outcomes of sleep questionnaire. Sleep 2009;32:915-9.
42 Weaver TE, Laizner AM, Evans LK, et al. An instrument to measure functional status outcomes for disorders of excessive sleepiness. Sleep 1997;20:835-43.

43 Johns MW. A new method for measuring daytime sleepiness: the epworth sleepiness scale. Sleep 1991;14:540-5.

44 McMillan A, Bratton DJ, Faria R, et al. Continuous positive airway pressure in older people with obstructive sleep apnoea syndrome (predict): a 12-month, multicentre, randomised trial. Lancet Respir Med 2014;2:804-12.

45 Versteegh M, Knies S, Brouwer W. From good to better: new Dutch guidelines for economic evaluations in healthcare. Pharmacoeconomics 2016;34:1071-4.

46 Husereau D, Drummond M, Petrou S, et al. Consolidated health economic evaluation reporting standards (cheers) statement. BMJ 2013;346:f1049.

47 Sullivan SD, Mauskopf JA, Augustovski F, et al. Budget impact analysis-principles of good practice: report of the ISPOR 2012 budget impact analysis good practice II Task force. Value Health 2014;17:5-14.

48 Elze MC, Gregson J, Baber U, et al. Comparison of propensity score methods and covariate adjustment: evaluation in 4 cardiovascular studies. J Am Coll Cardiol 2017;69:345-57.

49 Zaremba S, Mojica JE, Eikermann M. Perioperative sleep apnea: a real problem or did we invent a new disease? F1000Res 2016;5. doi:10.12688/f1000research.7218.1. [Epub ahead of print: 11 Jan 2016].

50 Nepomnayshy D, Hesham W, Erickson B, et al. Sleep apnea: is routine preoperative screening necessary? Obes Surg 2013;23:287-91.

51 de Raaff CAL, Coblijn UK, de Vries N, et al. Is fear for postoperative cardiopulmonary complications after bariatric surgery in patients with obstructive sleep apnea justified? A systematic review. Am J Surg 2016;211:793-801.

52 George CF. Reduction in motor vehicle collisions following treatment of sleep apnoea with nasal CPAP. Thorax 2001;56:508-12. 